\title{
La Suède et le Neutre chez deux auteurs franco- maghrébins contemporains : vers une nouvelle utopie ambiguë?
}

Maria Walecka-Garbalinska

Université de Stockholm

Si le Nord a de tout temps fasciné les voyageurs et les écrivains français, rares sont les romans dont l'action se déroule sous ces latitudes ${ }^{\mathrm{I}}$. Il est d'autant plus intéressant de voir la Scandinavie émerger, vers la fin des années I980, comme espace romanesque dans la littérature francomaghrébine. Mohammed Dib, Algérien écrivant en France, fait paraître entre 1985 et 1994 une tétralogie nordique saluée par la critique $^{2}$; le Marocain Abdelkébir Khatibi, traducteur des écrivains suédois, publie en 1990 un roman autobiographique intitulé Un été à Stockholm. Quelques années plus tard, Tahar Ben Jelloun, lauréat du prix Goncourt de 1987, envoie en Suède un des narrateurs de son roman Le dernier $a m i$. La question qui se pose d'emblée est celle des raisons et des enjeux d'un décentrement référentiel à première vue inattendu chez des auteurs qui, ancrés dans deux cultures et deux langues, sont d'une façon ou d'une autre concernés par les problèmes particuliers de "l'intellectuel colonisé-décolonisé »(Khatibi, I97I : I8I). Cette question sera abordée à travers l'articulation de trois concepts élaborés dans différents contextes théoriques, mais complémentaires : ceux du Neutre, du Tiers espace et de l'utopie ambiguë. En nous concentrant sur les romans de Khatibi et de Ben Jelloun, qui évoquent la Suède de façon explicite, nous voudrions proposer une lecture selon laquelle cet espace géoculturel fonctionne dans leur écriture comme écran de projection d'un désir du Neutre proche de celui décrit par Roland Barthes, tout en rejoignant par certains côtés les poncifs de l'imaginaire du Nord traditionnel. Dans le contexte francophone, une certaine utopie scandinave pourrait dès lors être interprétée comme l'équivalent imaginaire et symbolique

How to cite this book chapter:

Walecka-Garbalinska, M. 20I5. La Suède et le Neutre chez deux auteurs francomaghrébins contemporains : vers une nouvelle utopie ambiguë ? In: Cedergren, M. et Briens, S. (eds.) Médiations interculturelles entre la France et la Suède. Trajectoires et circulations de 1945 à nos jours. Pp. 33-43. Stockholm: Stockholm University Press. DOI: http://dx.doi.org/Io.I6993/bad.d. License: CC-BY 
du tiers espace d'énonciation postcoloniale, permettant de dépasser les binarismes culturels figés et d'articuler la différence culturelle au-delà de l'opposition du Même et de l'Autre.

\section{Territoires du Neutre ${ }^{3}$}

Le terme de "Neutre » renvoie au titre du cours de sémiologie littéraire que Roland Barthes a donné au Collège de France en 1977-78 et dont

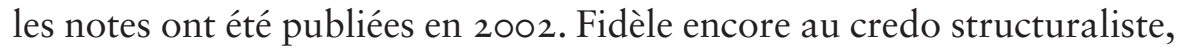
Barthes y transfère la catégorie linguistique du genre grammatical sur le terrain du discours, entendu comme "tout syntagme articulé par le sens ", donc textes, conduites socialement codées, " motions intérieures du sujet "(Barthes, 2002 : 216). Selon sa définition, relève du Neutre " toute inflexion qui esquive ou déjoue la structure paradigmatique, oppositionnelle, du sens, et [qui] vise par conséquent à la suspension des données conflictuelles du discours"(Barthes, 2002 : 26I-262). Ces inflexions du sens sont décrites par Barthes à travers une vingtaine de «figures » (ou «traits »), telles que le Silence, la Bienveillance, la Fatigue, l'Oscillation, etc., en opposition à celles de l'Anti-Neutre : l'Arrogance, la Colère, l'Adjectif. Parmi celles-là certaines peuvent servir à rendre compte des représentations littéraires de la Suède dans les romans des écrivains convoqués ici.

La «suspension des données conflictuelles du discours » (ibid.) c'est également ce qui, dans le domaine plus restreint de la critique postcoloniale, est l'objectif de Homi K. Bhabha lorsqu'il lance son concept clé de « tiers espace » d'énonciation. Celui-ci serait « la condition préalable à toute articulation de la différence culturelle ", ouvrant " la voie à la conceptualisation d'une culture fondée non pas sur l'exotisme du multiculturalisme ou la diversité des cultures, mais sur l'inscription et l'articulation de l'bybridité de la culture » dont la signification est élaborée dans l'entre-deux de la traduction et de la négociation. L'exploration du tiers espace devrait ainsi permettre, selon Bhabha, d' " éluder la politique de polarité et enfin émerger comme les autres de nous-mêmes. » (Bhabha, 2007 : 83. Les italiques sont de Bhabha).

Le tiers espace a inévitablement une dimension utopique en tant qu'il signifie une dialectique brisée de la modernité et une mise en valeur de l'ambiguïté. À ce propos Bhabha cite Walter Benjamin pour qui «l'ambiguïté est l'apparence figurative de la dialectique, la loi de la dialectique au point mort ». Et d'ajouter : "Pour Benjamin, cet arrêt est Utopie [...] » (Bhabha, 2007 : 54). Or, c'est en conjuguant ces mêmes termes d'utopie 
et d'ambiguïté que Vincent Fournier décrivait, dans l'ouvrage éponyme, la vision moderne de la Scandinavie comme "lieu des puretés et des vertus » (Fournier, I989: 294) naturelles qui garantissaient une résolution miraculeuse des contradictions historiques et des tensions sociales. L'auteur montre comment cette vision, formulée de façon canonique par les voyageurs romantiques français au XIX ${ }^{\mathrm{e}}$ siècle, est ensuite réactivée au contact des idéologies différentes, généralement conservatrices, au cours de la période précédant la Première guerre mondiale. Il analyse en particulier le dispositif rhétorique à l'œuvre dans le discours sur la Scandinavie, qui, avec la théorie des climats et ses différentes réinterprétations comme fondement, repose sur le transfert des propriétés physiques du paysage vers le terrain de l'anthropologie ${ }^{4}$.

Dans les romans en question le déplacement des personnages "sudistes » vers le Nord devient l'occasion d'une prise de conscience des clivages et de l'entre-deux de différentes natures : linguistique, identitaire, culturelle, existentielle. Il génère en même temps une tentative de leur dépassement ou de leur suspension dans laquelle l'espace et la culture suédois, perçus comme intermédiaires, indécidables et radicalement différents, jouent un rôle important. L'alter ego de Khatibi dans Un été à Stockholm se rend dans la capitale suédoise en tant qu'interprète pour un colloque sur la neutralité et y fait l'expérience d'une " extase froide »: entre deux avions, deux femmes et deux langues. Dans Le dernier ami de Ben Jelloun, l'histoire de l'amitié tourmentée de deux Tangerois se déroule sur le fond de l'évolution de la société marocaine depuis la fin des années cinquante jusqu'à la fin des années quatre-vingt-dix. La Suède, où l'un des deux amis s'exile - pour en revenir à la fin du livre - apparaît comme l'envers idéalisé d'un Maroc trop compliqué, mais finalement indispensable.

\section{Figures du Neutre scandinave}

Un des points communs qui dominent dans la quête identitaire ou existentielle des personnages romanesques "diasporiques ", c'est la fascination - ou la stupéfaction - face à l'impossibilité de placer la Suède dans les paradigmes établis. L'un d'eux explique : "Mais pourquoi la Suède ? Un pays neutre, à la fois européen et plus ou moins autre chose. - C'est cela qui m'excite : ce plus ou moins » (Khatibi, I990a : 72). Cependant, ce n'est que chez Khatibi que le Neutre est explicitement et littéralement évoqué comme une qualité intrinsèque de l'homme et du sol suédois. Son personnage-narrateur parle, par 
exemple, de "la beauté du neutre et de l'esprit de transparence » (Khatibi, I990a : 89) qui règnent en Suède et discute la question de la neutralité politique du pays. En tant qu'interprète, il traduit un long discours consacré à ce sujet, incorporé in extenso dans le roman ${ }^{5}$. Mais l'association entre le Nord et le Neutre se fait avant tout à travers différentes " figures », dont celle, fondamentale, du Silence.

\section{Le Silence et l'Oscillation}

Gérard Namir, le double narratif de Khatibi, remarque que les Suédois partagent avec les Japonais " une passion extraordinaire pour le silence » et il est vite subjugué par le "[s]ilence presque chantant » (Khatibi, I990a : 88) de son initiatrice suédoise Lena. Mais il ne s'agit pas seulement de la rareté de la parole. Le silence prend surtout une dimension sociale et correspond à un mode d'être ensemble moralement et esthétiquement valorisé, comme chez Ben Jelloun, dont le personnage rapporte à son ami resté au Maroc : "La première chose qu'on remarque quand on arrive en Suède, c'est le silence. Une société silencieuse, sans agitation, sans désordre. "(Ben Jelloun, 2004 : Io8) Le silence, omniprésent dans les descriptions de l'espace naturel et social scandinave, réunit l'homme et la nature dans un rapport d'analogie et de réversibilité ${ }^{6}$. Qualité immanente du Nord, le silence en fait l'emblème, la métonymie et la métaphore du Neutre, objet d'un désir et d'une éthique qui travaillent le discours romanesque maghrébin à l'instar de celui des poètes, des philosophes et des mystiques analysés par Barthes. En tant que jugement suspendu - ni oui ni non - le Silence déjoue, en effet, le paradigme du sens et ses oppositions binaires et rend possible la traduction culturelle dans le sens de Bhabha. Une Suède neutre et silencieuse pourrait représenter, pour le sujet postcolonial bilingue, cet espace à partir duquel il prend la parole au-delà des dualités qui déterminent sa position culturelle.

C'est ce qui se passe, en effet, dans la scène de l'interprétation simultanée au colloque sur la neutralité à Stockholm dans le récit de Khatibi. Dans sa cabine transparente - telle une parenthèse de l'arrêt du jugement - Gérard Namir adopte lui-même une stratégie neutre et s'applique à la discrétion professionnelle dans un va-et-vient impassible : "Je suis successivement moi-même, l'autre, et de nouveau moi-même, entre la vitesse et la parole, la vitesse et le silence. Dès que je suis excité, je me calme. »(Khatibi, I990a : 49). Pour le traducteur maghrébin l'usage d'une tierce langue signifie la suspension 
des oppositions binaires et d'un choix impossible : linguistique, émotionnel, identitaire. À travers cette activité, la différence culturelle est articulée hors du paradigme conflictuel, comme Oscillation, elle aussi figure du Neutre réhabilitée par Barthes : «l'oscillation, l'hésitation, l'alternance [...] accomplissent un temps vibré » et sont des moyens d'arriver à la justesse et à l'authenticité dans le rapport aux autres et à soi-même (Barthes, 2002 : I74).

\section{Le climat et l'anthropologie}

Les ingrédients obligés de l'imaginaire du Nord, comme la neige et le froid, sont également interprétés comme manifestations du Neutre et ont partie liée avec le silence selon la même logique discursive que Vincent Fournier a repérée dans les récits de voyage du XIX ${ }^{\mathrm{e}}$ siècle et qui consiste à construire l'être scandinave à l'image de son milieu naturel dont il serait le produit et l'émanation. Le froid et la neige mettent la sourdine sur les expressions de la subjectivité et les élans de sensibilité : " De cette rue me parvenait un murmure froid, telle une voix ouatée de neige " (Khatibi, I990a : 45), note le flâneur citadin de Khatibi qui admire le "calme neutre» (Khatibi, I990a : I43) propice au recueillement et l' "ambiance translucide " (Khatibi, I990a : 89) de Stockholm. Qualités qu'il attribue justement à la vertu de la neige qui « accorde à l'homme, cet homme, un extraordinaire désir de transparence " (Khatibi, I990a : 58). D’où le rêve " de se sentir transfiguré par la nature et ses anciens esprits, tel une sculpture runique, polie par les vents enneigés » (ibid.). Aussi, le moment culminant de son parcours initiatique sera-til celui d'une "extase froide" (Khatibi, I990a : 59) qui lui permettra de transcender l'opposition entre la rationalité froide occidentale (représentée dans le livre par Descartes mort de froid à Stockholm) et la spiritualité mystique ardente faisant partie de son héritage musulman. Une révélation en accord avec "la beauté de ce pays silencieux » et " pensif ", où les gens semblent " assis dans un temple ", dans " un effort de concentration sur soi, selon un rite de dévotion énigmatique » (Khatibi, I990a : 23, 26).

L'idée d'une spiritualité non-dogmatique, qui n'exclut ni la modernité ni un fonctionnement rationnel de la société, se retrouve également dans le récit de Ben Jelloun dont le héros est enthousiaste devant la perspective de quitter un Maroc, où il a connu la montée de l'intégrisme religieux et la persécution de l'opposition, pour aller en Suède. "J'allais vivre dans un autre monde, un univers étrange et passionnant, 
une société minée par l'angoisse métaphysique, mais une société éminemment évoluée » (Ben Jelloun, 2004 : I08-ı09), se disait-il.

\section{Bergman médiateur}

Il est intéressant de constater qu'à la source de cette représentation, il y a chez les deux auteurs marocains (et chez leurs personnages) les premiers films d'Ingmar Bergman dont ils s'étaient régalés dans les années soixante-dix avant même de visiter la Suède ${ }^{7}$. Pour ses admirateurs franco-marocains, Bergman devient le passeur vers un univers du silence (titre de son film de I963) et son cinéma véhicule l'image d'" un mode de vie particulier : la passion d'effacer les traces ", qui serait le sens profond du mot "neutralité ", selon l'interprétation khatibienne (Khatibi, I990a : 58, italique de l'auteur). Comme mode de vie et comme disposition naturelle, la neutralité se manifeste sur des plans divers de la vie collective et individuelle: "dans la mobilisation sociale, dans la différence entre les sexes, âges, groupes, quartiers, professions, sans oublier le cérémonial, le goût pour les rites de passage et les offrandes des fleurs avec des larmes retenues " (Khatibi, I990a : 88) ${ }^{8}$. On retrouve dans cette énumération proposée par le narrateur d'Un été à Stockholm plusieurs autres traits du Neutre étudiés par Barthes (dont Khatibi connaissait bien les travaux) : Retenue, Non-palmarès, Androgyne, rite comme dispositif de régulation dans l'ordre affectif (Barthes, 2002 : 163). Le médecin marocain dans Le dernier ami les relève également, d'une manière tout aussi explicite : "Le silence et la blancheur de la peau, les yeux clairs et le regard distant, le geste précis et rare, la politesse systématique, le respect des règles ", " [p]as de tapes dans le dos, ni d'embrassades ", " chaque individu a autant d'importance qu'un autre» (Ben Jelloun, 2004 : I08-109).

\section{Utopie ambiguë revisitée}

On pourrait multiplier les citations qui situent dans l'espace-temps scandinave le royaume du Neutre (modéré, bienveillant, adouci, etc.), sur les plans, interchangeables, de la nature, de l'ethnotype, de l'organisation sociale et politique. La réalisation historique du Neutre, dont la Suède serait le lieu exemplaire, fait apparaître en creux (chez Khatibi) ou explicitement (chez Ben Jelloun) l'anti-Neutre de leur société d'origine : arrogance du pouvoir, violation des droits de l'homme, hiérarchies artificielles, anarchie quotidienne. Le personnage du Dernier ami 
le formule ainsi : «[...] je regardais ce pays avec mes yeux de Marocain et de médecin qui avaient tant souffert du manque de respect de la personne et du manque de rigueur d'une société qui ne fait que s'arranger» (Ben Jelloun, 2004 : I08).

En tant qu'objet de désir et moyen de critique, le modèle suédois relève clairement de l'utopie dans l'écriture romanesque maghrébine. Désigné souvent comme un là-bas indéfini, comme un univers étrange, ou encore comme un espace des limites ultimes, du jour et nuit confondus, incolore et inodore, ne produisant même pas de poussière, un pays où les gens se nourrissent de peu et mangent froid (Ben Jelloun, 2004 : I IO), ce monde dématérialisé apparaît dans un éloignement quasi-mythique renforcé par le fantasme insulaire omniprésent. Le double narratif de Khatibi, qui séjourne sur l'île de Skeppsholmen au cœur de la capitale suédoise, s’imagine être dans un : « [s]ite inspiré en quelque sorte par une féerie luthérienne rigoureuse, mise sous glace» (Khatibi, I990a : 37). Le quartier de la vieille ville, par sa situation isolée ( "blottie sur une île»), interstitielle et mouvante ("ville entre les ponts » qui « est un récit de voyage ", Khatibi, I990a : 87), devient à son tour la métaphore spatiale d'un entre-deux existentiel et culturel riche de promesses initiatiques. Les significations analogues de temps suspendu et de jouissance autarcique, propres au fantasme utopique, réapparaissent aussi dans le motif de la fête de midsommar sur une île de l'archipel.

La Suède est une société fascinante, certes, mais reste pour les voyageurs maghrébins un ailleurs impossible. C'est pourquoi, une fois leur initiation achevée, ils quittent le paradis enneigé pour retrouver une temporalité historique. L'enthousiasme initial de l'exilé de Tanger tourne en mal du pays : "C'est idiot, mais ce qui me manquait le plus, c'étaient des choses qui m'énervaient comme le bruit des voisins, les cris des vendeurs ambulants, les pannes d'ascenseur [...] me manquait aussi la poussière »(Ben Jelloun, 2004: I09). Sans que jamais diminue son admiration pour " ce pays magnifique " (I2O) et ses habitants qui sont, dit-il, " de vrais musulmans bons, généreux, solidaires » (I 20) ; il retourne cependant mourir au pays, à cause des cimetières peut-être, qui dans le Nord sont « froid(s), rangé(s), triste(s)» (I47).

Dans la diégèse, le déplacement vers le Nord, tout en étant un éblouissement, s'associe souvent pour les voyageurs maghrébins à une expérience de l'échec ou de la perte (de la santé, de l'amitié, de la famille) qui change le fonctionnement textuel des figures du Neutre. Dans le récit de Ben Jelloun, c'est par délicatesse ${ }^{9}$ que Mamed, parti travailler en Suède, tait à son meilleur ami qu'il est atteint d'un cancer 
dangereux. Le silence et la discrétion - manifestations du Neutre qui esquive l'assertion - deviennent mensonge et détruisent leur relation. À Stockholm, sous le choc du diagnostic qu'il vient de recevoir, l'homme entre dans un bar et se confie à un inconnu qui lève son verre sans un mot de réconfort. Mamed apprécie d'une certaine façon ce geste de délicatesse qui évite de réduire le chagrin de l'autre "à un cas qui relève[rait] très normalement d'une explication ou d'une classification générale »(Barthes, 2002:67), mais c'est à Tanger qu'il trouvera apaisement, auprès de son vieux père qui lui explique que la Suède est un modèle encore lointain.

Dans Un été à Stockholm, le thème de l'ambiguïté du Neutre est annoncé dès le début du récit à travers le discours sur la neutralité traduit en simultané par le narrateur au cours d'une conférence internationale. Tout en exposant les avantages de la neutralité politique, l'orateur se demande s'il est possible de « tout neutraliser - la guerre et la paix, les sexes, les classes, les familles, les excès de la consommation, de la pollution et de la pauvreté ?»(Khatibi, I990a : 57). L'idylle scandinave en tant que laboratoire d'un projet utopique et ambigu se dévoile dans le dernier chapitre qui peut être lu comme une vision d'avenir effrayante. Il évoque notamment la vie d'une famille solitaire et alcoolique, dont l'existence parfaitement réglée dans "un foyer de cristal » (Khatibi, I990a : I6I) en banlieue de Stockholm, avec une assurance sociale jusqu'à la fin des temps » (Khatibi, I990a : I67), ressemble à un hivernage dans un silence glacial, où l'on vit et meurt par procuration devant la télévision. Toutes les manifestations du Neutre : la neige qui estompe le bruit, le froid qui modère les tensions sociales, le silence pyrrhonien, basculent alors dans une étrange dystopie postmoderne ${ }^{\mathrm{Io}}$. L'année même de la parution d'Un été à Stockholm Khatibi a employé, dans un entretien avec Ben Jelloun, le concept du «neutre »d'une façon qui éclaire cette ambiguïté, sinon cette contradiction. Discutant la technique dans le contexte global, il se demande : "va-t-elle libérer le monde de toute théologie, ou au contraire, va-t-elle permettre un servage [...], sous la suprématie de nouvelles totalités non moins absolues : l'État omnipuissant, la technocratie, le neutre. » Et il insiste par la suite sur ce dernier terme précisément, pour lui donner une acception fort différente de celle que lui conférait Barthes :

Oui, le neutre comme triomphe d'une impersonnalité monumentale effaçant jusqu'au corps sensible de l'homme et jusqu'à ses sensations les plus intimes : oui, le neutre comme existence d'une classe universelle amorphe, 
asservie aux problèmes de la consommation et de la survie, le neutre comme dévastation lente et lentement illimité de l'homme ; voilà ce qui nous attend peut-être. (Khatibi, I990b : I I 8).

\section{Conclusion}

Force est de constater que la représentation de l'espace scandinave qui se dégage des textes littéraires franco-maghrébins convoqués a des points de contacts avec celle que Fournier a dégagée chez les voyageurs-essayistes français de la fin du millénaire précédent. Les contenus et les enjeux ne sont évidemment pas les mêmes dans l'écriture postcoloniale à l'ère de la mondialisation. Il ne s'agit plus d'une utopie régressive teintée de rousseauisme, ni de synthèse du primitif et du social fantasmée dans le cadre d'une pensée de l'exotisme et du nationalisme montant. Bien au contraire, le détour par le Nord conduit les narrateurs homodiégétiques et étrangers professionnels ${ }^{\mathrm{II}}$ à la reformulation de l'identité individuelle et à la prise de conscience de leur extranéité, de la part " la moins méditerranéenne » (Khatibi, I999:49) de leur mémoire. L'espace suédois configuré comme neutre devient ainsi l'équivalent imaginaire du Tiers espace comme moyen de "se libérer du cercle vicieux de la dialectique coloniale» (Scharfman, I990: 74) qui pour l'intellectuel francophone ne finit pas avec la décolonisation. Comme le dit Ronnie Scharfman : " [l]'idéal est de la dépasser entièrement, tout en reconnaissant une ambivalence foncière qui laissera toujours des traces. » (Scharfman, I990: 74). Le Nord neutre pourrait bien traduire cet idéal et cette ambivalence et dans ce sens représenter un nouvel avatar de l'utopie ambiguë, construite toujours à travers la rhétorique de l'échange entre la nature et l'homme, le paysage et la société, les espaces du dehors et du dedans.

\section{Notes}

I. On peut consulter à ce sujet Dubar, M. \& Moura, J.-M. (éd.) (2000), Le Nord, latitudes imaginaires. Presses de l'Université Charles-de-GaulleLille 3, coll. "UL3 travaux et recherches "; Fournier V. (200I), Le voyage en Scandinavie : anthologie de voyageurs I627-I9I4. Paris : Laffont, coll. "Bouquins » et Andersson, K. (éd.) (2004-2007), L’image du Nord chez Stendhal et les Romantiques I-IV. Örebro Universitetsbibliotek, coll. "Humanistica Oerebroensia. Artes et linguæ».

2. Aux titres qui figurent dans la bibliographie, il faut ajouter Le sommeil d'Ève de 1989. 
3. Nous réservons la majuscule à cette forme substantivée uniquement. Dans les citations, l'orthographe originale sera évidemment respectée.

4. Dans ce volume, l'article de Jean-François Battail est consacré à l'archéologie de ces représentations.

5. Selon son propre aveu dans La langue de l'autre, la question de la neutralité politique passionnait Khatibi : "mon texte a misé sur l'adhésion de la Suède à l'Union Européenne, trois ans avant que ce soit fait. [...] [J]'ai étudié avec maints détails le paradoxe de la neutralité. C'est une chose incompréhensible pour la plupart des États, sinon des peuples. Un pays en paix perpétuelle, est-ce possible ? Que signifie cette neutralité absolue ? " (Khatibi, I999: 84). Sur la neutralité comme stratégie énonciative d'effacement des traces culturelles chez Khatibi, cf. Moustir (2013).

6. Aussi la narratrice de l'Infante maure de Mohammed Dib affirme que « les lacs [de la Finlande] c'est du silence fait eau, lumière ", alors que la montagne, emblématique du paysage familier de son père maghrébin, " c'est un grand bruit énorme » (Dib, I994: I7I). Lorsqu'elle cherche la réconciliation de ces deux territoires entre lesquels elle est déchirée, c'est toujours au silence qu'elle se réfère : "La neige produit le silence, le sable aussi produit le silence » (Dib, I994 : I 5 I). Cf. Bonn (2002).

7. Khatibi avoue d'ailleurs avoir conçu son roman en sous-titrant en français les films de Bergman : " Je sous-titrais les dialogues et les visions, les voix de cette langue étrangère [...], dans le respect de son timbre, son rythme, son silence, dans cette impression inoubliée de la neige par laquelle la passion d'effacer des traces me brûlait les mains " (Khatibi, I999: 64).

8. Allusion probable aux réactions à l'assassinat d'Olof Palme en 1986.

9. La délicatesse est une des figures du Neutre analysées par Barthes qui résume : "J'appellerai volontiers le refus non violent de la réduction, l'esquive de la généralité par des conduites inventives, inattendues, non paradigmatisables, la fuite élégante et discrète devant le dogmatisme, bref le principe de délicatesse, je l'appellerai en dernière instance : la douceur. " (Barthes, 2002:66).

Io. Elle n'est pas sans rappeler la vision de Mohammed Dib dans ses Terrasses d'Orsol dont le héros erre à travers une ville vaguement scandinave, moderne et opulente, mais hantée par la présence d'une fosse innommable où grouillent de monstrueuses créatures, exclues de la cité idéale.

I I. Gérard Namir, à l'instar de son créateur, se voit comme un "voyageur professionnel qui veut traverser les frontières avec une souplesse d'esprit » (Khatibi, I990a: 9). 


\section{Bibliographie}

Barthes, R. (2002), Le Neutre. Notes de cours au Collège de France 1977I978, texte établi, annoté et présenté par Clerc, T. Paris : Seuil/IMEC.

Ben Jelloun, T. (2004), Le dernier ami. Paris : Seuil.

Bhabha, H. K. (2007), [1994], Les lieux de la culture. Une théorie postcoloniale (trad. de l'anglais par Bouillot, F). Paris : Payot.

Bonn, C. (2002), "La steppe, le désert, la neige : fonctions de l'absence ", in Khadda, N. (éd.), Mohammed Dib 50 ans d'écriture. Université de Montpellier III. Disponible en ligne : http://www.limag.com/Textes/Bonn/ DibDesertNaget.htm

Dib, M. (1985), Terrasses d'Orsol. Paris, Sindbad.

— (1990), Neiges de marbre. Paris : Sindbad.

— (1994), L'infante maure. Paris : Albin Michel.

Fournier, V. (I989), L'utopie ambiguë. La Suède et la Norvège chez les voyageurs et essayistes français (I882-I9I4). Clermont-Ferrand: Adosa.

Khatibi, A. (I97I), La mémoire tatonée. Paris : Denoël.

— (I990a), Un été à Stockholm. Paris : Flammarion.

— (I990b), " Entretien avec Tahar Ben Jelloun ", in Cheng, F. et al., Abdelkebir Khatibi. Rabat : Édition Okad : I I 5-I 26.

- (I999), La langue de l'autre. New York-Tunis : Éditions Les Mains Secrètes.

Moustir, H. (20I3), « De l'intersémiotique au neutre narratif et identitaire dans l'œuvre romanesque de Khatibi ", in Expressions maghrébines (vol.I2), I : I9-37.

Scharfman, R. (I990), "Autobiographie maghrébine : La Mémoire Tatouée de Abdelkébir Khatibi » in Cheng, F et al. Abdelkebir Khatibi. Rabat : Édition Okad : 6I-76. 